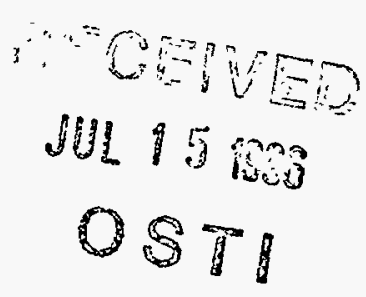

Toy Simulation of ATLAS SUSY Points*

\author{
Frank Paige \\ Brookhaven National Laboratory \\ Upton, New York 11973, USA
}

* ATLAS Physics Note ATLAS-PHYS-No-085

This manuscript has been authored under contract number DE-AC02-76CH00016 with the U.S. Department of Energy. Accordingly, the U.S. Government retains a non-exclusive, royalty-free license to publish or reproduce the published form of this contribution, or allow others to do so, for U.S. Government purposes. 


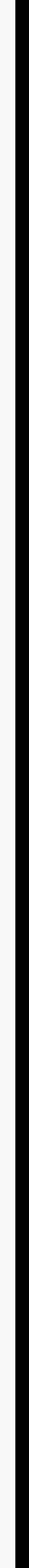




\section{DISCLAIMER}

This report was prepared as an account of work sponsored by an agency of the United States Government. Neither the United States Government nor any agency thereof, nor any of their employees, makes any warranty, express or implied, or assumes any legal liability or responsibility for the accuracy, completeness, or usefulness of any information, apparatus, product, or process disclosed, or represents that its use would not infringe privately owned rights. Reference herein to any specific commercial product, process, or service by trade name, trademark, manufacturer, or otherwise does not necessarily constitute or imply its endorsement, recommendation, or favoring by the United States Government or any agency thereof. The views and opinions of authors expressed herein do not necessarily state or reflect those of the United States Government or any agency thereof. 



\title{
Toy Simulation of ATLAS SUSY Points
}

\author{
Frank E. Paige \\ Physics Department \\ Brookhaven National Laboratory \\ Upton, NY 11973 \\ 24 April 1996
}

\begin{abstract}
The five points in supersymmetry parameter space being studied by ATLAS are examined using a toy detector simulation.
\end{abstract}

\section{Event Simulation}

The minimal supergravity model is based on the minimal supersymmetric extension of the standard model together with grand unification and radiative electroweak symmetry breaking. It is characterized by a common scalar mass $m_{0}$, a common gaugino mass $m_{1 / 2}$, and a common trilinear SUSY breaking parameter $A_{0}$ at the GUT scale, a ratio $\tan \beta$ of vacuum expectation values at the weak scale, and $\operatorname{sgn} \mu= \pm 1$. This is known as the SUGRA model in ISAJET. While this model has obvious appeal because of its simplicity and approximate consistency with the precision electroweak data from LEP and SLC, one should remember that it covers only a subset of possible SUSY models.

ATLAS has decided to study five points in the SUGRA model, as described in Table 1 below. The numbering of the points is arbitrary. Point 1 was selected to be consistent with a reasonable amount of cold dark matter using the calculation of Falk and Olive. Point 4 is one of the points also being studied for the NLC. A top mass of $170 \mathrm{GeV}$ was used for the signal; the default $180 \mathrm{GeV}$ was used for the background. While this is annoying, it is probably not significant.

Table 1: SUGRA parameters and some representative masses.

\begin{tabular}{|c|c|c|c|c|c|c|c|c|c|c|}
\hline Point & $\begin{array}{c}m_{0} \\
(\mathrm{GeV})\end{array}$ & $\begin{array}{c}m_{1 / 2} \\
(\mathrm{GeV})\end{array}$ & $\begin{array}{c}A_{0} \\
(\mathrm{GeV})\end{array}$ & $\tan \beta$ & $\operatorname{sgn} \mu$ & $\begin{array}{c}M_{\tilde{g}} \\
(\mathrm{GeV})\end{array}$ & $\begin{array}{c}M_{\tilde{u}_{R}} \\
(\mathrm{GeV})\end{array}$ & $\begin{array}{c}M_{\tilde{W}_{1}} \\
(\mathrm{GeV})\end{array}$ & $\begin{array}{c}M_{\bar{e}_{R}} \\
(\mathrm{GeV})\end{array}$ & $\begin{array}{c}M_{h} \\
(\mathrm{GeV})\end{array}$ \\
\hline 1 & 100 & 300 & 300 & 2.1 & + & 767 & 663 & 230 & 157 & 100 \\
\hline 2 & 400 & 400 & 0 & 2.0 & + & 1004 & 926 & 324 & 430 & 107 \\
\hline 3 & 400 & 400 & 0 & 10.0 & + & 1009 & 928 & 318 & 431 & 122 \\
\hline 4 & 200 & 100 & 0 & 2.0 & - & 300 & 313 & 97 & 207 & 68 \\
\hline 5 & 800 & 200 & 0 & 10.0 & + & 586 & 912 & 117 & 805 & 115 \\
\hline
\end{tabular}


This note describes some general properties of the signal events for these points using ISAJET 7.17 plus a toy detector simulation based on CALSIM. Version 7.17 differs from the released 7.16 only in that the top mass is properly included in the $W+t$ and $Z+t$ cross sections. The calorimeter energy resolutions are taken to be

$$
\begin{aligned}
\text { EMCAL } & 10 \% / \sqrt{E}+1 \% \\
\text { HCAL } & 50 \% / \sqrt{E}+3 \% \\
\text { FCAL } & 100 \% / \sqrt{E}+7 \%,|\eta|>3
\end{aligned}
$$

A uniform segmentation $\Delta \eta=\Delta \phi=01$. is used with no transverse shower spreading. This is particularly unrealistic for the forward calorimeter. Jets are found using GETJET with a fixed cone size $R=0.7$, and missing energy is found from the calorimeter. Leptons and $B$ hadrons are taken from the generator. All these quantities are saved in a DST for further analysis.

A sample of $10 \mathrm{~K}$ events was generated for each point, corresponding to integrated luminosities of $0.56 \mathrm{fb}^{-1}, 3.1 \mathrm{fb}^{-1}, 3.2 \mathrm{fb}^{-1}, 7.5 \mathrm{pb}^{-1}$, and $0.33 \mathrm{fb}^{-1}$ respectively. A sample of $50 \mathrm{~K}$ events for each of $t \bar{t}, W j$ with $W \rightarrow e \nu, \mu \nu, \tau \nu$, and $Z j$ with $Z \rightarrow \nu \bar{\nu}, \tau \tau$ was generated in five bins covering $50<p_{T}<1600 \mathrm{GeV}$. The background samples correspond to luminosities ranging from $4.3 \mathrm{pb}^{-1}$ in the lowest $p_{T}$ bin of $W j$ up to more than $100 \mathrm{fb}^{-1}$ for the highest $p_{T} Z j$ bin. QCD jet backgrounds require both much more statistics and a simulation of the detector incorporating realistic resolutions - including in particular any cracks or other non-Gaussian tails - and capable of processing $>10^{6}$ events in a reasonable time. Hence QCD backgrounds have not been studied. Past experience suggests that they are likely to be relevant only for fairly low masses, e.g., for Point 4.

\section{Properties of SUSY Events}

Fig. 1 shows the distribution of SUSY particles produced in the primary interactions using the ISAJET codes, which are listed in the caption. Note that wino and zino production often competes with gluino and squark production even at LHC. It is important to generate everything.

Fig. 2 for each sample shows the missing energy $\mathbb{E}_{T}$ calculated from summing all the cells of the toy calorimeter. Fig. 3 shows the difference in $\mathbb{F}_{T}$ calculated from the the sum of all cells and from the sum of calorimeter clusters with $E_{T}>10 \mathrm{GeV}$. The difference is small and seems to be quite Gaussian. Summing such clusters could be useful to reduce the effect of pileup at high luminosity, since pileup events generally do not contain jets. A more realistic simulation including, e.g., transverse shower spreading in the forward calorimeter, is needed to understand this.

Fig. 4 shows the transverse sphericity $S_{T}$, i.e, the ratio $2 \lambda_{2} /\left(\lambda_{1}+\lambda_{2}\right)$ of eigenvalues of the sphericity tensor in the transverse plane. Since gluinos and squarks are produced with $p_{T} \sim M$, one expects them to give "round" events, so a cut $S_{T}>0.2$ is typically made.

Fig. 5 shows the inclusive jet $p_{T}$ distribution for central jets having a jet axis with $|\eta|<3$. (Leptons are also found as jets by GETJET; these have been removed by matching isolated leptons with jets.) Fig. 6 shows the $p_{T}$ distribution for the three hardest 
central jets. The second peak at low $E_{T}$ for Point 5 is caused by chargino and neutralino production. These are relatively light and so have large cross sections even though they are produced via electroweak couplings. Fig. 7 shows the multiplicity of jets with $E_{T}>50$, 100 , and $200 \mathrm{GeV}$. Some of these soft jets may represent tails of harder jets in the events, but it is clear that the events are quite complicated.

Fig. 8 shows the multiplicity distribution for leptons with $p_{T}>10 \mathrm{GeV},|\eta|<2.5$, and satisfying the isolation requirement $E_{T}<5 \mathrm{GeV}$ in $R=0.2$. Fig. 9 shows the $p_{T}$ distribution for the three hardest such leptons.

Fig. 10 shows the multiplicty of $b$ jets, tagged by having a $B$ hadron with $p_{T}>20 \mathrm{GeV}$ and $\eta<2$ within $R=0.3$ of the jet. These cuts are intended to ensure that the tracks from the $B$ are reasonably hard and are in the acceptance of the tracker. However, no inefficiency or false tags are included. Fig. 11 shows the $p_{T}$ spectrum of such jets. For high masses it is necessary to be able to tag $b$ jets up to $\mathcal{O}(1 \mathrm{TeV})$, since the multiplicity and distribution of such jets may help to determine the SUSY model and parameters. Understanding the ability to do this requires a rather detailed study of pattern recognition for tracks in jets.

If the decay $\tilde{Z}_{2} \rightarrow \tilde{Z}_{1} h$ is kinematically allowed, it is generally large. In many cases is possible to observe the light Higgs $h$ decaying into $b \bar{b}$ pairs, although in some cases there is too much combinatorial background even with a perfect detector. Fig. 12 shows the distribution of dijet masses tagged as $b$ jets together with a Gaussian plus linear fit. The light Higgs is visible for Points $1-3$, the ones for which the decay is allowed. The fitted masses are a few GeV low but track the actual Higgs masses (for this perfectly linear and perfectly calibrated calorimeter). Fig. 13 shows the distribution of generated light Higgs bosons. From this figure one can clearly see the relatively large rates for $h$ for the first three points compared to the last two. For large $p_{T}$ the two $b$ jets may merge. Also, the $b$ jets from such events are boosted $50 \mathrm{GeV}$ jets, so they have lower multiplicity and smaller opening angles than inclusive $b$ jets at the same $p_{T}$. Thus both inclusive and $h \rightarrow b \bar{b}$ (or $Z \rightarrow b \vec{b})$ samples should be considered when studying $B$ tagging at high $p_{T}$.

\section{Signals, Backgrounds, and Mass Estimates}

The basic signature for supersymmetry at the LHC is multiple jets plus $\mathbb{T}_{T}$. The prop erties of such events depend on the relationship among the various masses. For Points 1 , 2 , and 3 , the decay chain

$$
\begin{array}{r}
\tilde{g} \rightarrow \tilde{q}_{R} \bar{q} \\
\tilde{q}_{R} \rightarrow \tilde{Z}_{1} q
\end{array}
$$

is kinematically allowed and has a substantial branching ratio. This gives a large rate for two hard acollinear jets plus $\mathbb{F}_{T}$, similar to the signature recently used by D0 to search for $\tilde{t}_{1} \rightarrow \tilde{Z}_{1} c$. For Point 4 the light squarks are slightly heavier than the gluino. This case is not well treated by ISAJET, since the gluinos are decayed using phase space matrix elements ignoring the nearby poles. While $\tilde{g} \rightarrow \tilde{b} \bar{b}$ is allowed, the branching ratio for $\tilde{b} \rightarrow \tilde{Z}_{1} b$ is very small. For Point 5 the squarks are much heavier than the gluino. 
Table 2: The value of $M_{4 j}$ for which $S+B=2 B$ compared to $M_{\text {SUSY, }}$, the lighter of the gluino and squark masses. The cuts are too high compared to the masses for Point 4.

\begin{tabular}{|c|c|c|c|}
\hline Point & $M_{4 j}(\mathrm{GeV})$ & $M_{\text {SUSY }}(\mathrm{GeV})$ & Ratio \\
\hline 1 & 960 & 663 & 1.45 \\
\hline 2 & 1240 & 926 & 1.34 \\
\hline 3 & 1240 & 928 & 1.34 \\
\hline 4 & - & - & - \\
\hline 5 & 840 & 586 & 1.43 \\
\hline
\end{tabular}

Fig. 14 is designed to select $\tilde{q}_{R} \tilde{q}_{R}$ events, which should be important for Points 1-3. The events in this figure are required to have two jets with $E_{T}>100 \mathrm{GeV}, E_{T}>100 \mathrm{GeV}$, and $S_{T}>0.2$. Any third jet must have $E_{T, 3}<0.2 E_{T, 1}$, and any isolated lepton must have $p_{T, \ell}<20 \mathrm{GeV}$. Then the quantity

$$
M_{\mathrm{eff}, 2 j}=E_{T, 1}+E_{T, 2}+\mathbb{E}_{T}
$$

is plotted for the signal and for the $t \bar{t}, W j$, and $Z j$ backgrounds.

If the gluino is lighter than the squarks, as in Points 4 and 5 , then multijet events should dominate. Fig. 15 is designed to select these. The events in this figure are required to have at least four jets with $E_{T, 1}>100 \mathrm{GeV}, E_{T, 2,3,4}>50 \mathrm{GeV}, E_{T}>100 \mathrm{GeV}$, and $S_{T}>0.2$. Then the quantity

$$
M_{\mathrm{eff}, 4 j}=E_{T, 1}+E_{T, 2}+E_{T, 3}+E_{T, 4}+E_{T}
$$

is plotted for the signal and for the $t \bar{t}, W j$, and $Z j$ backgrounds.

For Points $1,2,3$, and 5 there is a clear break in the shape of the $M_{\text {eff, } 4 j}$ distribution. For Points $1,2,3$, there is also a pretty clear signal in the $M_{\text {eff }, 2 j}$ distribution, while for Point 5 with heavy squarks there is no $M_{\text {eff, } 2 j}$ signal above background. For Point 4 the

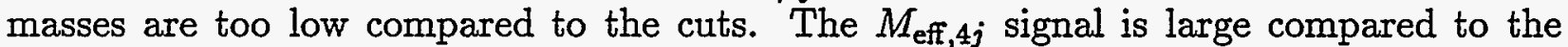
background everywhere, and the $M_{\text {eff }, 2 j}$ is not statistically clear. For this point the QCD background, which has been ignored here, is likely to be important.

Fig. 16 shows the IDENT codes of all the SUSY particles in the events passing the cuts in Fig. 14. Note there is multiple counting here; e.g., every event has two $\tilde{Z}_{1}$ 's. There is substantial enhancement of $\tilde{q}_{R}$ over $\tilde{q}_{L}$, but the latter is not negligible. Also there is a substantial rate for $\tilde{W}_{1}$. Without the lepton veto, the $\tilde{q}_{R}: \tilde{q}_{L}$ ratio was only about $2: 1$, so it may be that $\tilde{q}_{L} \rightarrow \tilde{W}_{1} q, \tilde{W}_{1} \rightarrow \tau \tilde{Z}_{1}$ is important. If so, a cut on low multiplicity jets might produce a purer sample. Unfortunately the DST does not contain information about $\tau$ 's or jet multiplicity.

The place where the $M_{4 j}$ signal deviates from the background can be used to estimate the SUSY masses. Since there are no peaks in Fig. 15, the point where the signal plus background exceeds the background by a factor of two, interpolated to the nearest half bin, will be used as an estimate of where the signal emerges from the background. This is 
clearly not optimal, since it uses the cross sections at one point rather than all the data. Nevertheless, it is compared with the mass of the lighter of the gluino and squarks in Table 2. At least over this limited sample the ratio is quite constant and would determine the mass to better than $10 \%$. Of course it is necessary to know the background for this to work, but the $t, W$, and $Z$ cross sections can be measured in other channels and compared with the distribution at lower values of $M_{4 j}$ to constrain it.

In addition to the multijet plus $\mathbb{E}_{T}$ signature there are many other singatures for supersymmetry. One important one is isolated like-sign dileptons $\ell^{ \pm} \ell^{ \pm}$. Since the gluino is a Majorana fermion, its branching ratios for the decay chains

$$
\tilde{g} \rightarrow \tilde{W}_{1}^{ \pm} X \rightarrow \ell^{ \pm} X
$$

are identical, whereas most standard model backgrounds give $\ell^{ \pm} \ell^{\mp}$ rather than $\ell^{ \pm} \ell^{ \pm}$. In Fig. 17, events with $T_{T}>100 \mathrm{GeV}, S_{T}>0.2$, and two isolated same-sign dileptons are selected using the lepton cuts described above. Then the quantity

$$
M_{\mathrm{eff}, 2 l}=p_{T, \ell 1}+p_{T, \ell 2}+E_{T, 1}+E_{T, 2}+E_{T, 3}+E_{T, 4}+E_{T}
$$

is plotted for the signal and the sum of the $t \bar{t}, W j$, and $Z j$ backgrounds. The Monte Carlo statistics are limited, but the place where $M_{\text {eff, } 2 l}$ deviates from the background seems to track the SUSY masses fairly well. Again the cuts are too low to treat Point 4 properly, and the QCD background is probably important for it. The coincidence of many such signatures will be essential to establish that the effect is due to supersymmetry. 

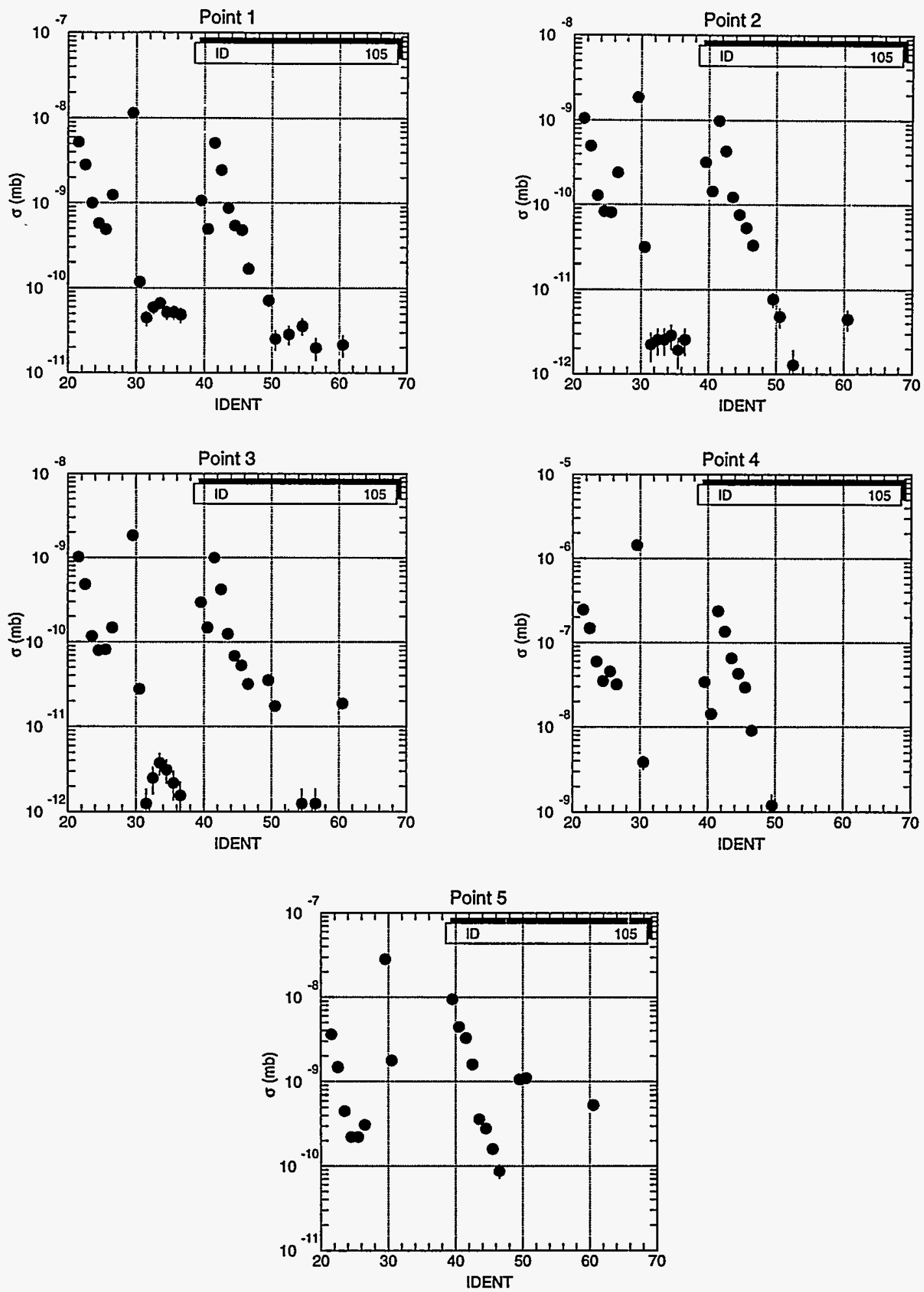

Figure 1: ISAJET types for SUSY particles produced in the primary processes: $\tilde{q}_{i, L}=21-26, \tilde{g}=29, \tilde{Z}_{1}=30, \tilde{\nu}_{i, L}, \tilde{\ell}_{i, L}=31-36, \tilde{W}_{1}=39$, $\tilde{Z}_{2}=40, \tilde{q}_{i, R}=41-46, \tilde{W}_{2}=49, \tilde{Z}_{3}=50, \tilde{\ell}_{i, R}=52,54,56, \tilde{Z}_{4}=60$. 

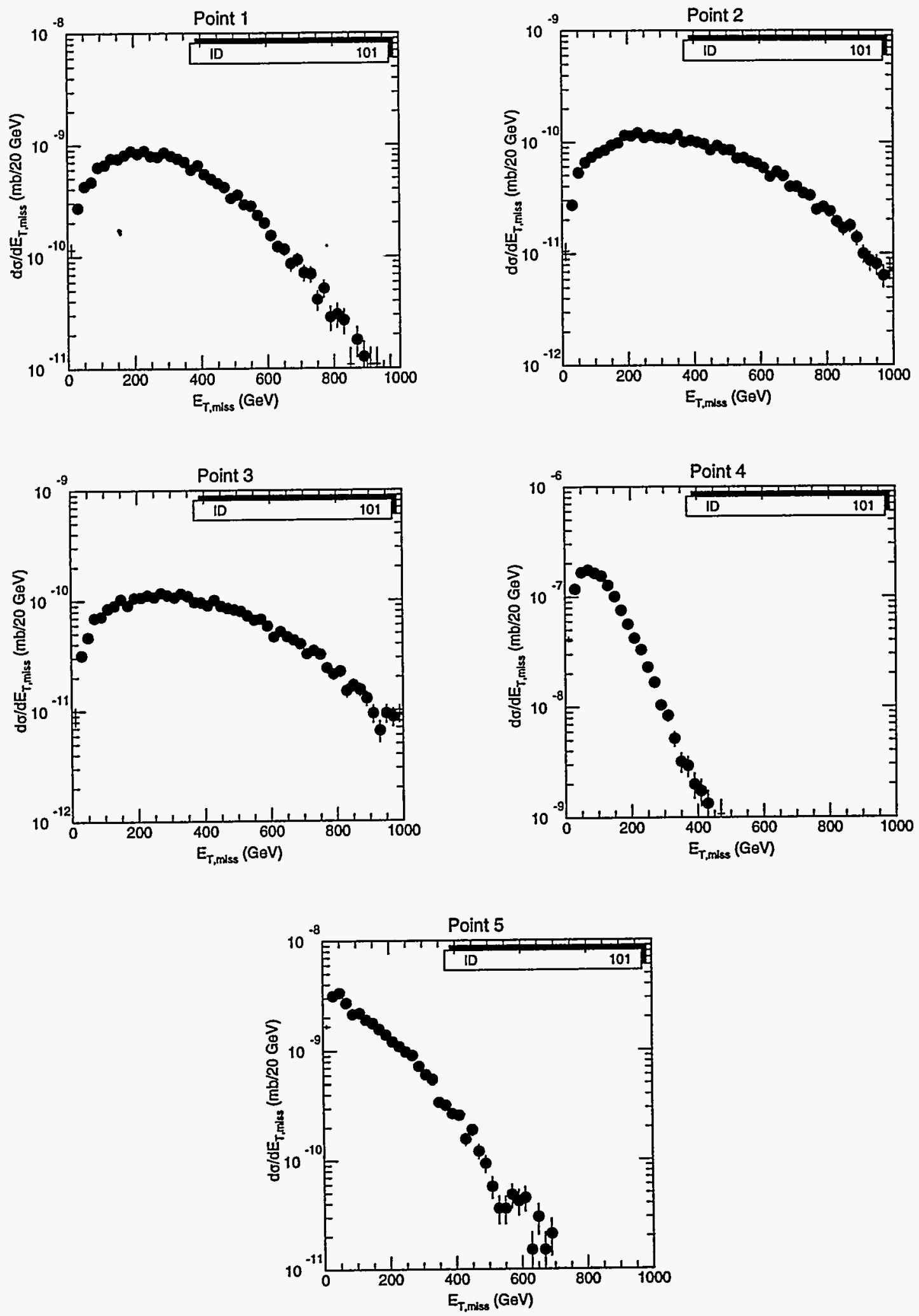

Figure 2: $T_{T}$ distributions. 

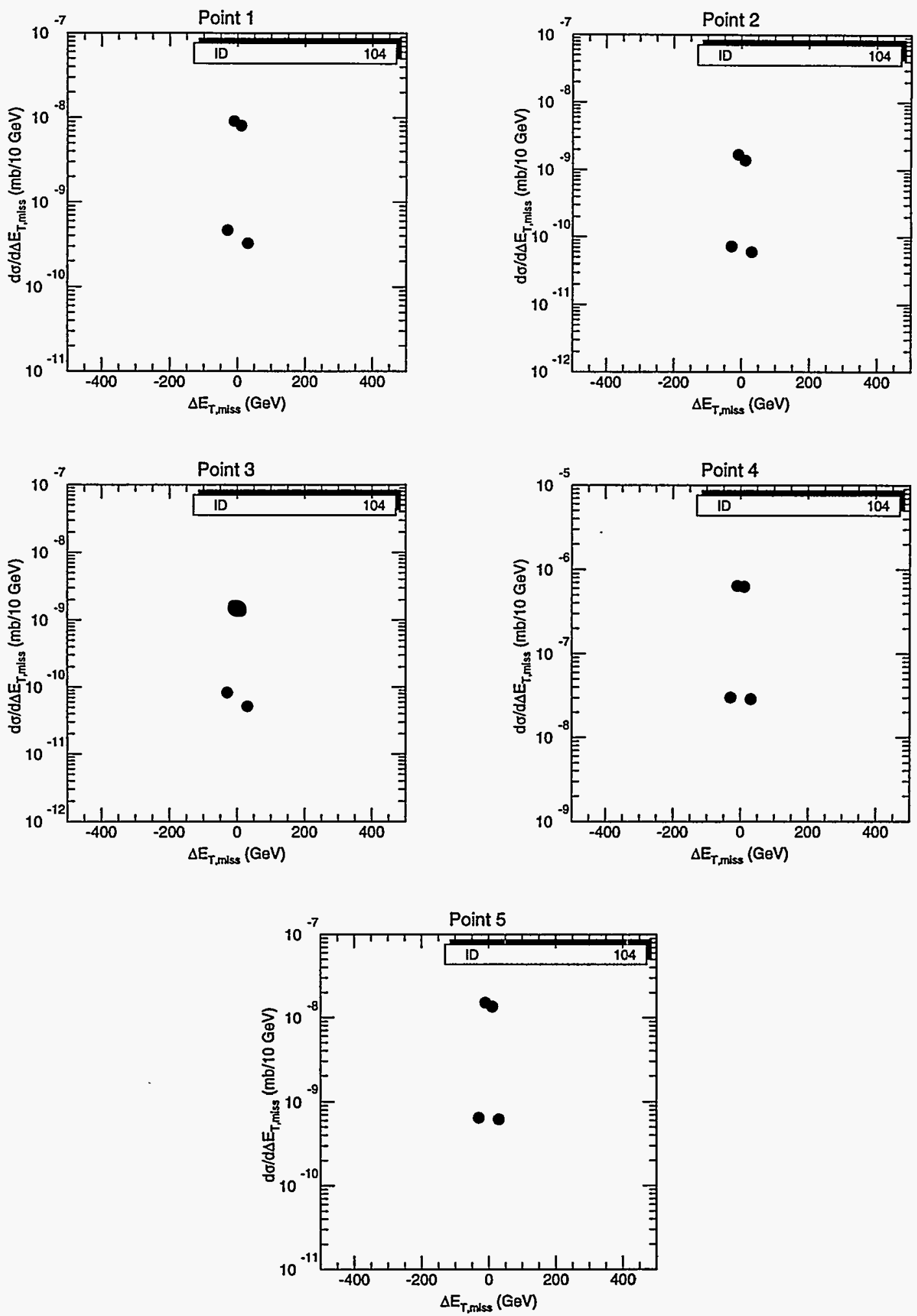

Figure 3: Differences in $\not_{T}$ using the full calorimeter and clusters with $E_{T}>10 \mathrm{GeV}$. 

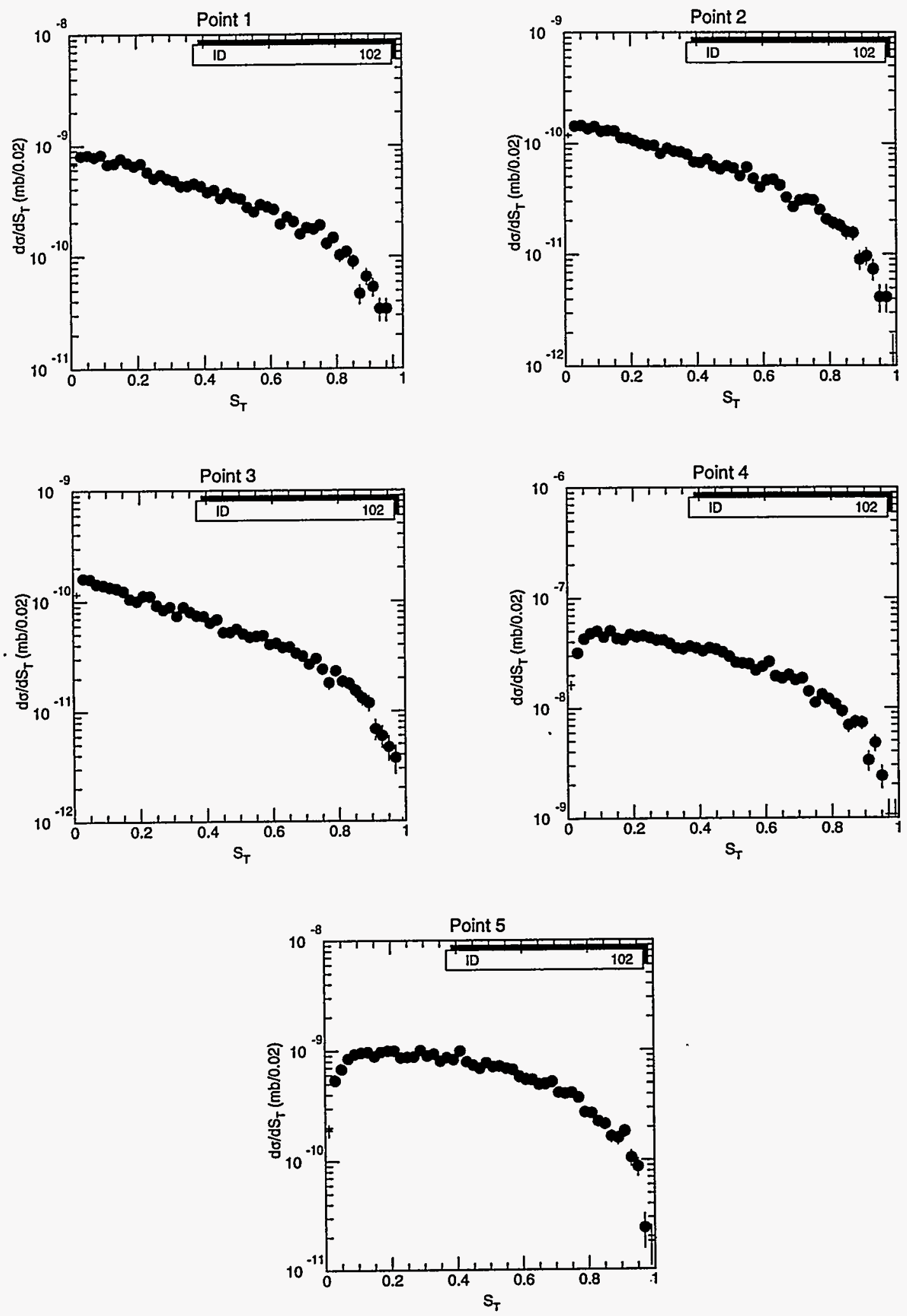

Figure 4: Transverse sphericity $S_{T}$ distributions. 

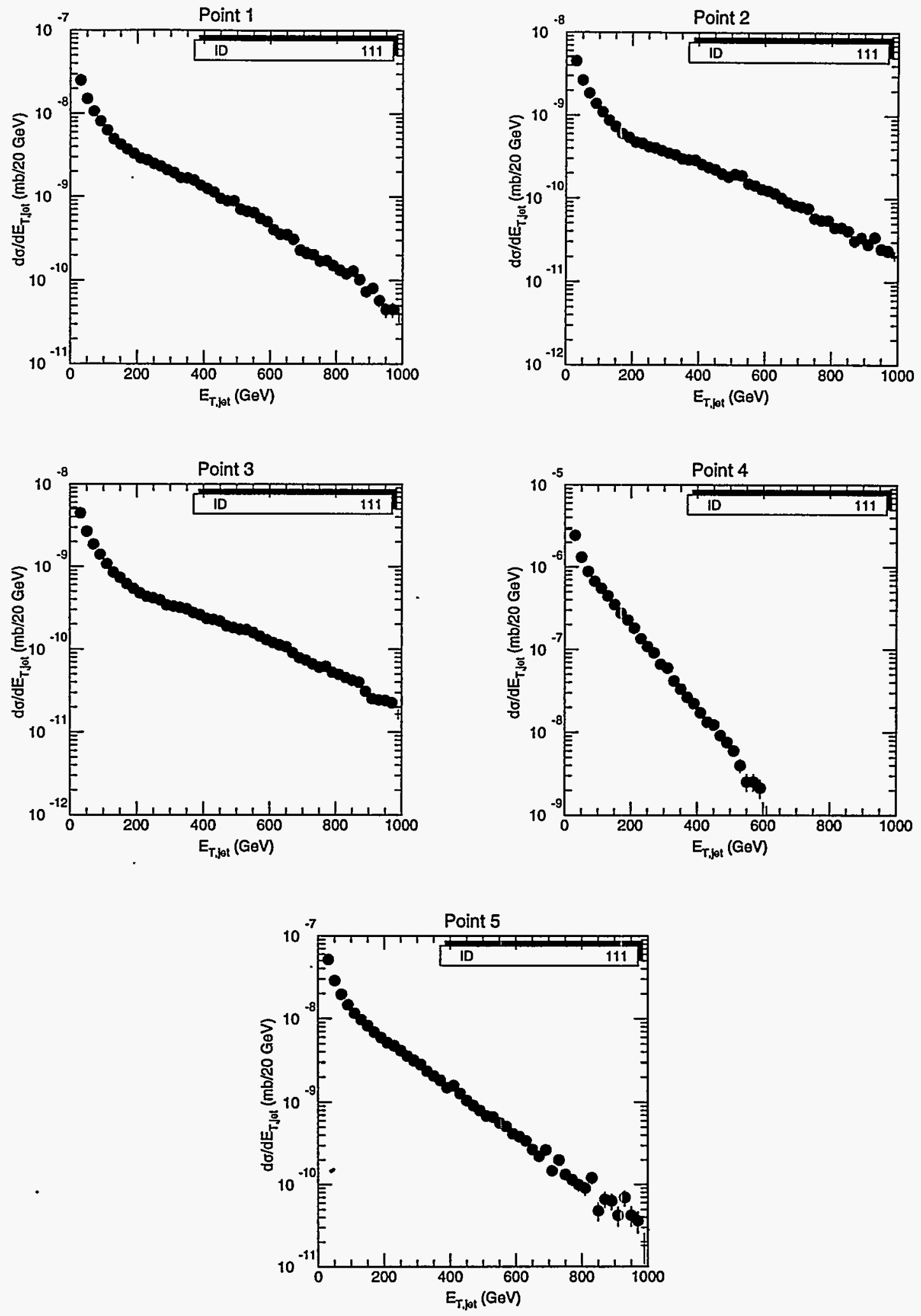

Figure 5: Central jet $p_{T}$ distributions. 

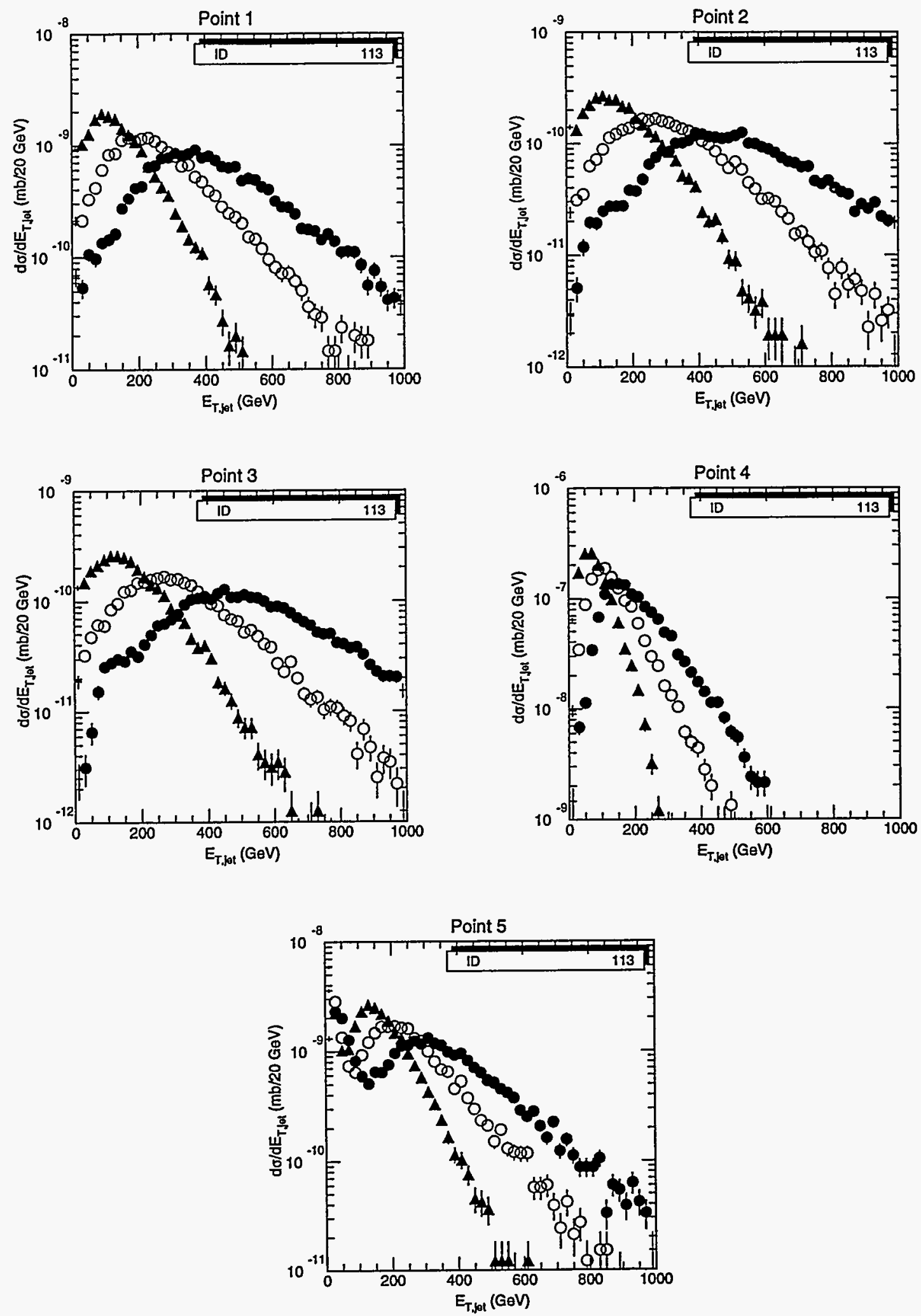

Figure 6: $p_{T}$ distributions for the three hardest jets. 

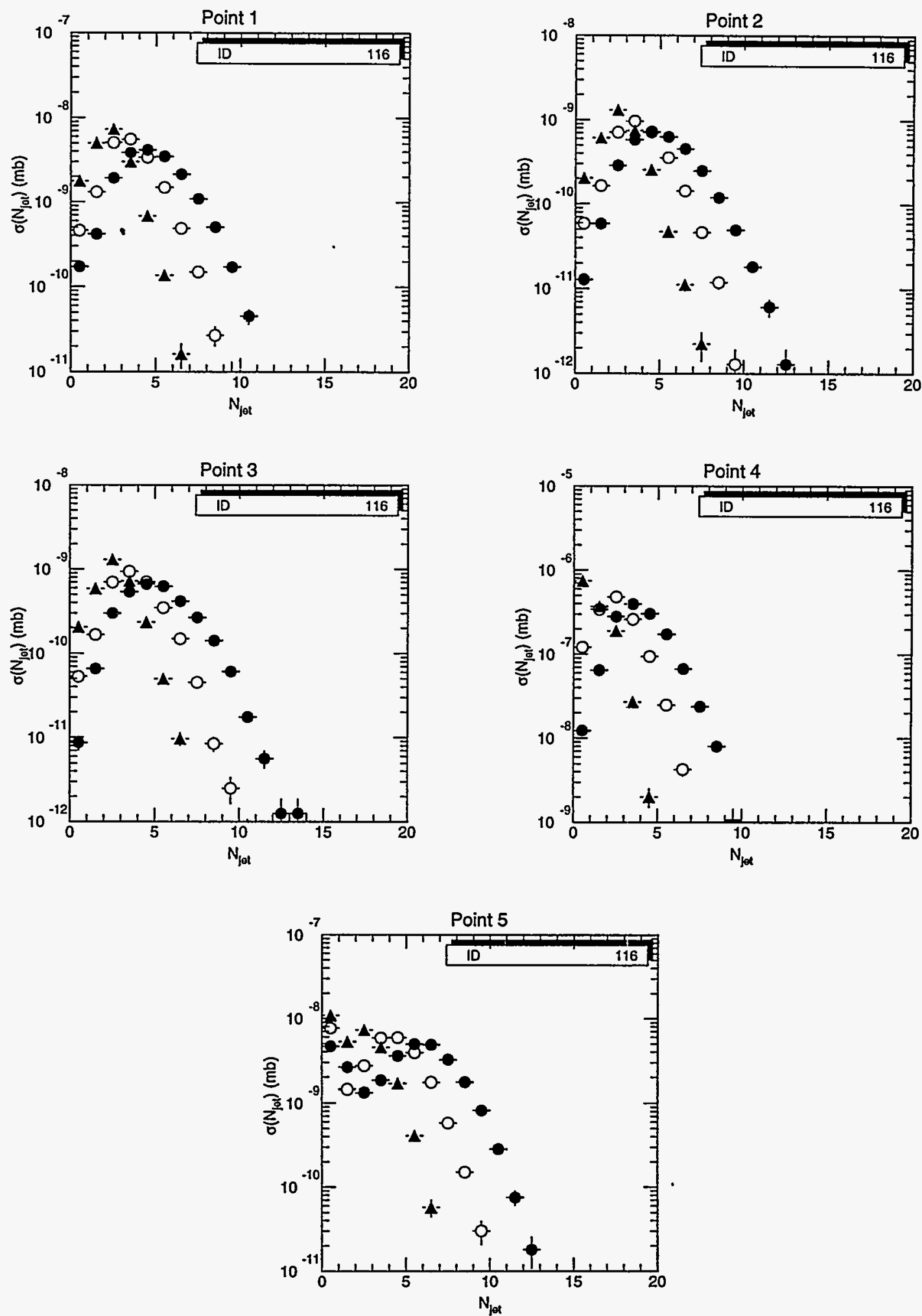

Figure 7: Multiplicity distributions for jets with $p_{T}>50 \mathrm{GeV}$ (circles), $100 \mathrm{GeV}$ (open circles), and $200 \mathrm{GeV}$ (triangles). 

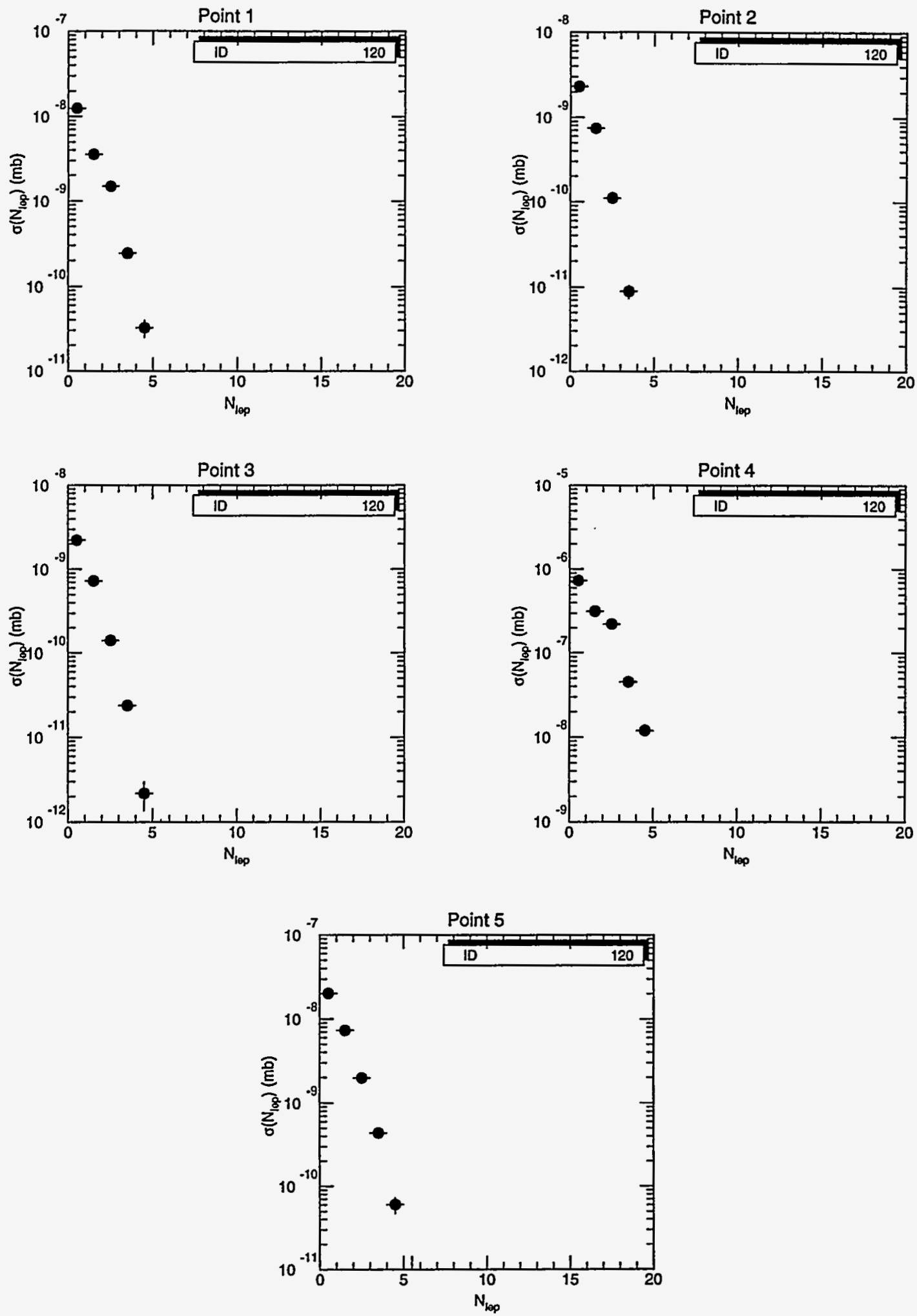

Figure 8: Multiplicity distributions for isolated leptons with $p_{T}>10$ and $|\eta|<3$. 

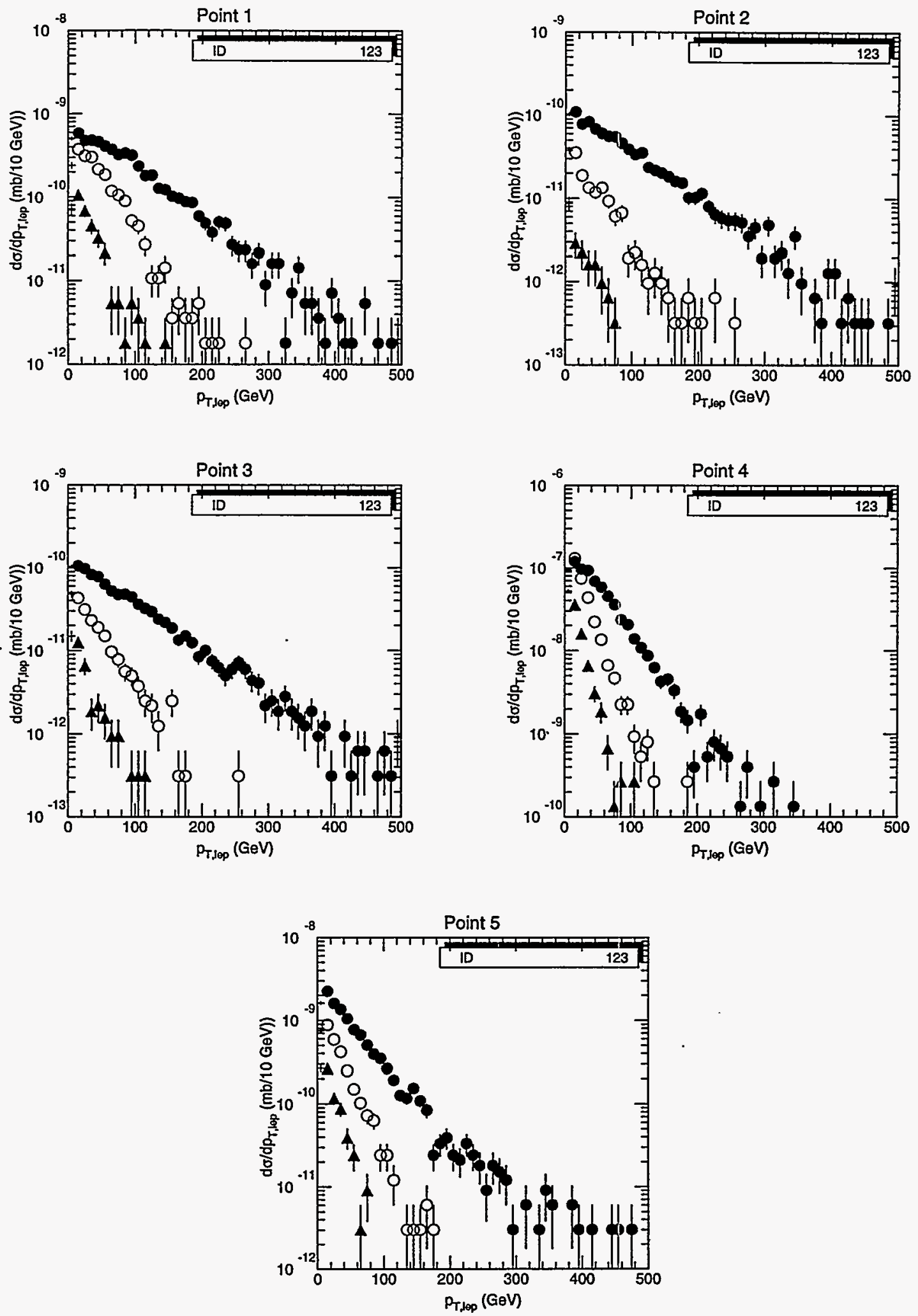

Figure 9: $p_{T}$ distributions for the three hardest leptons. 

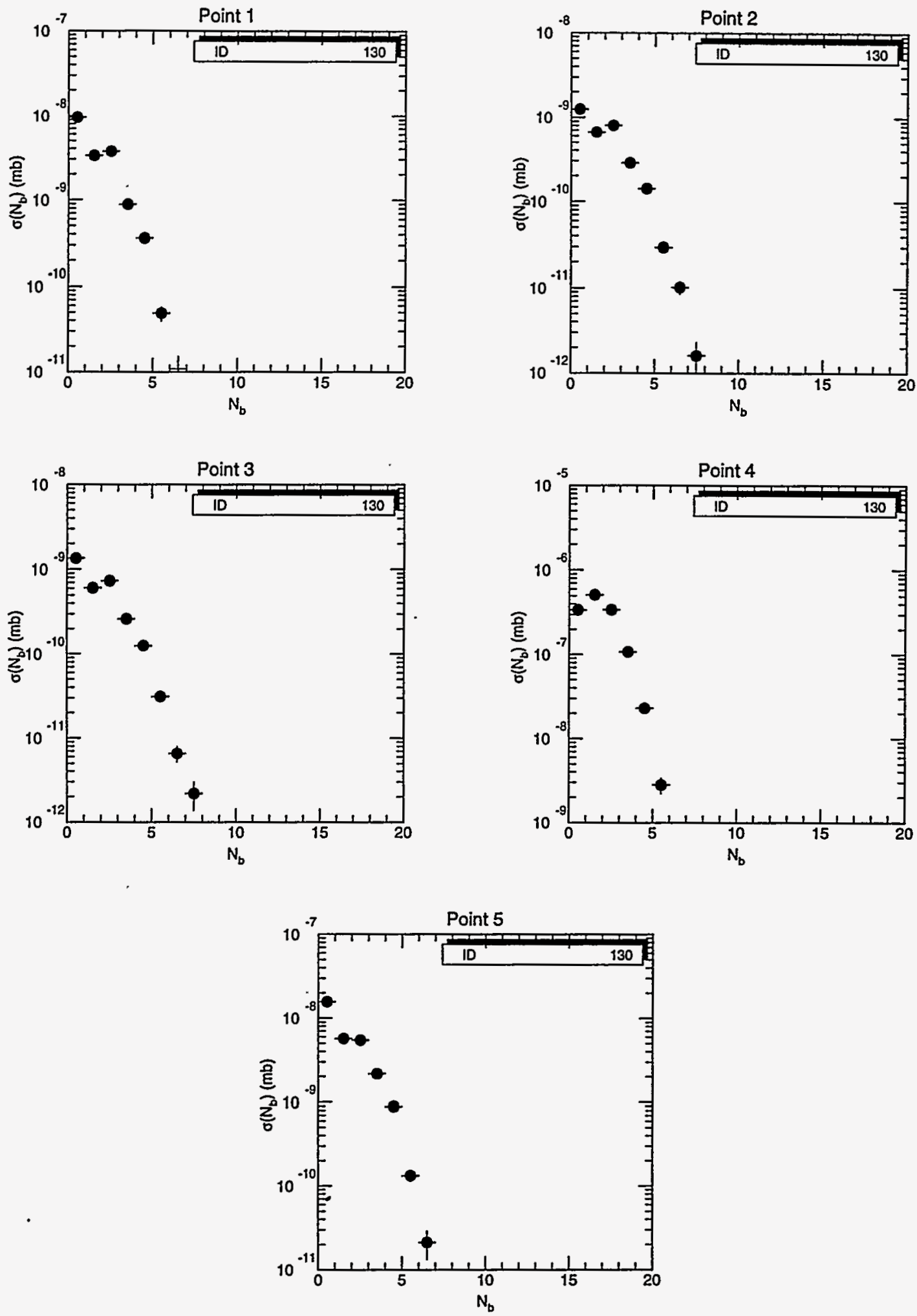

Figure 10: Multiplicty distributions for $B$ jets. Note that $\tilde{g} \rightarrow \tilde{b} \bar{b}$ dominates for Point 4 , accounting for its peak at $N_{b}>0$. 

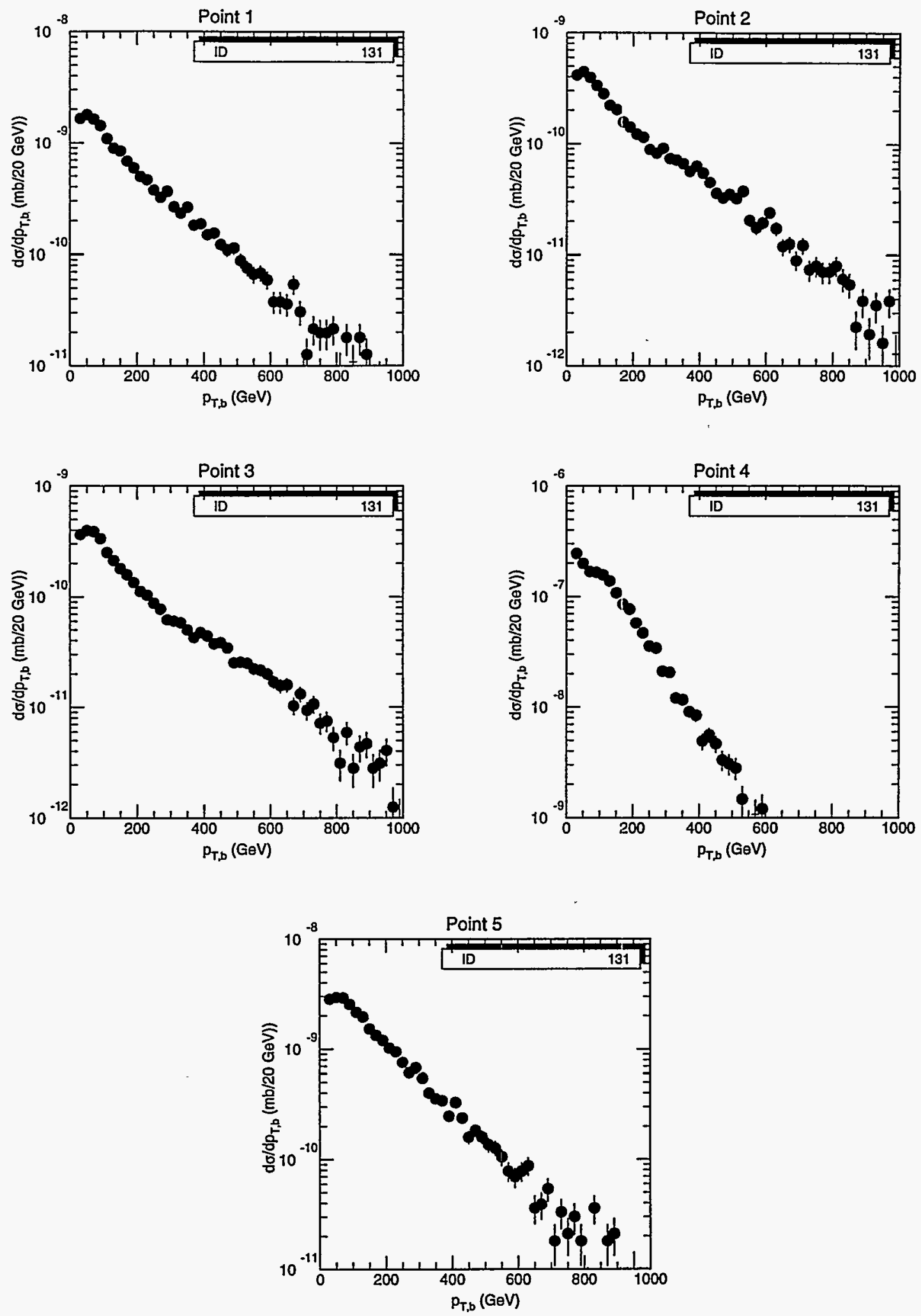

Figure 11: $p_{T}$ distributions for $B$ jets. 
Point 1

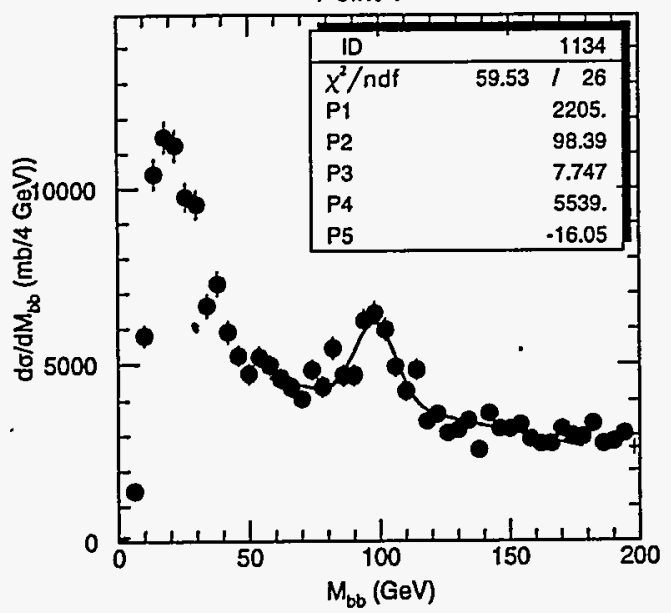

Point 3

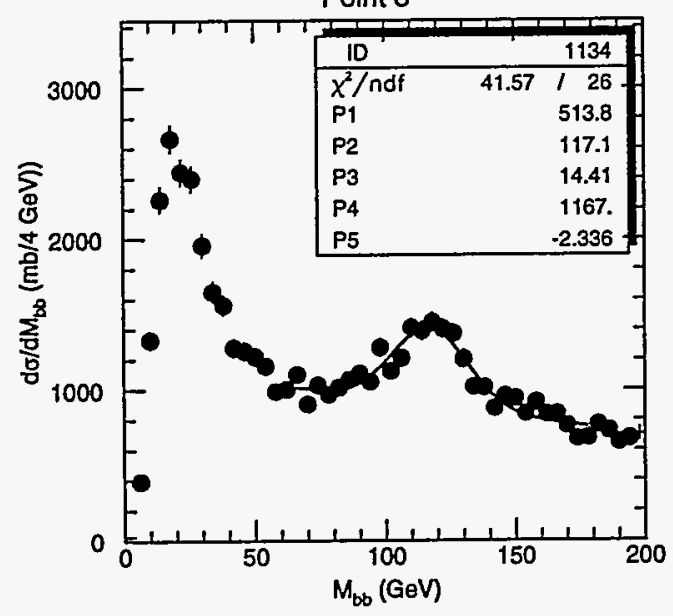

Point 2
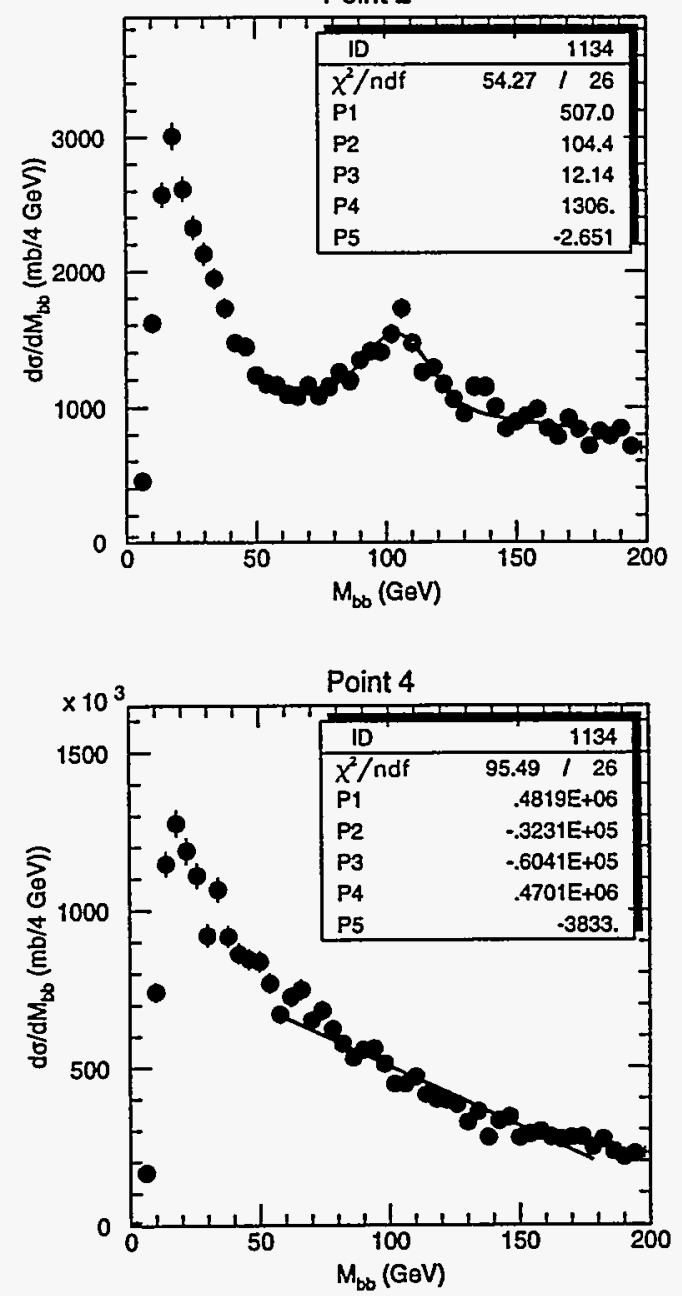

Point 5

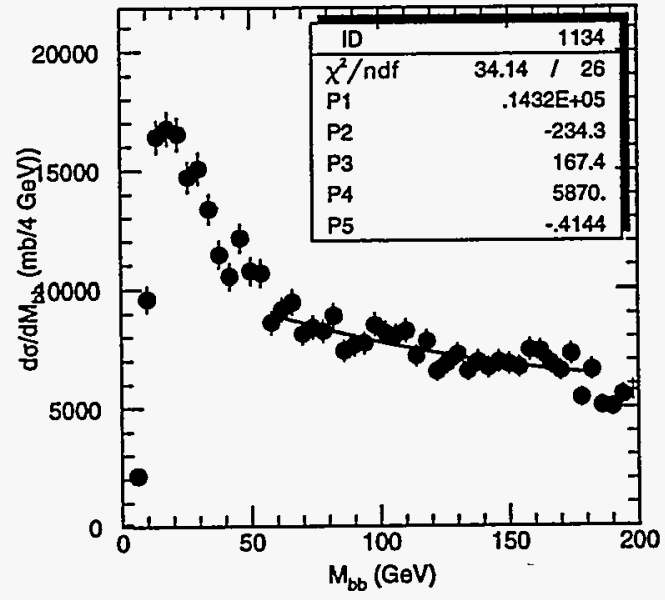

Figure 12: Mass distributions for pairs of $B$ jets together with a Gaussian plus linear fit. The fitted mass is P2 and should be compared with the last column in Table 1. 

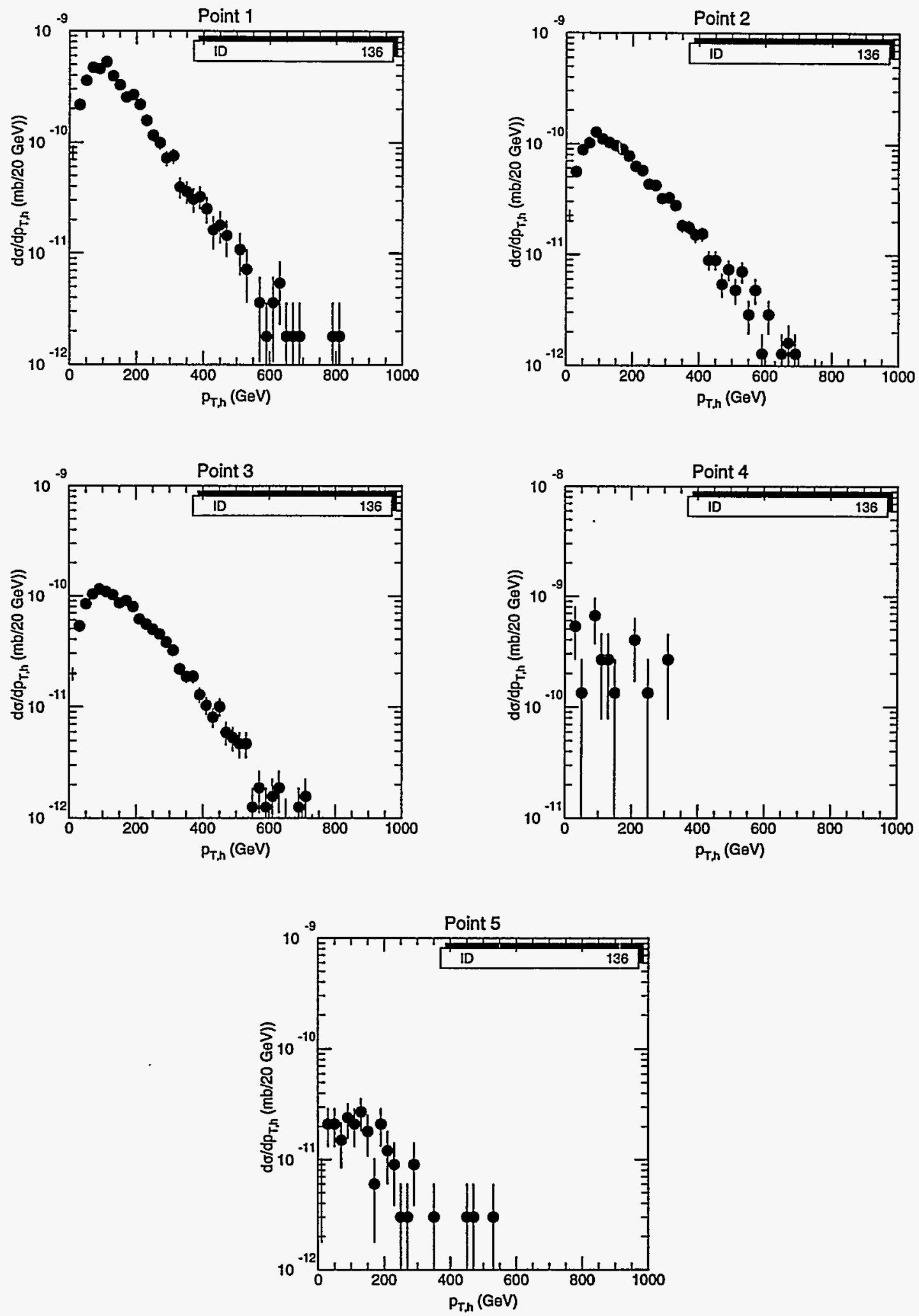

Figure 13: Generated $p_{T}$ distributions for light Higgs bosons. 

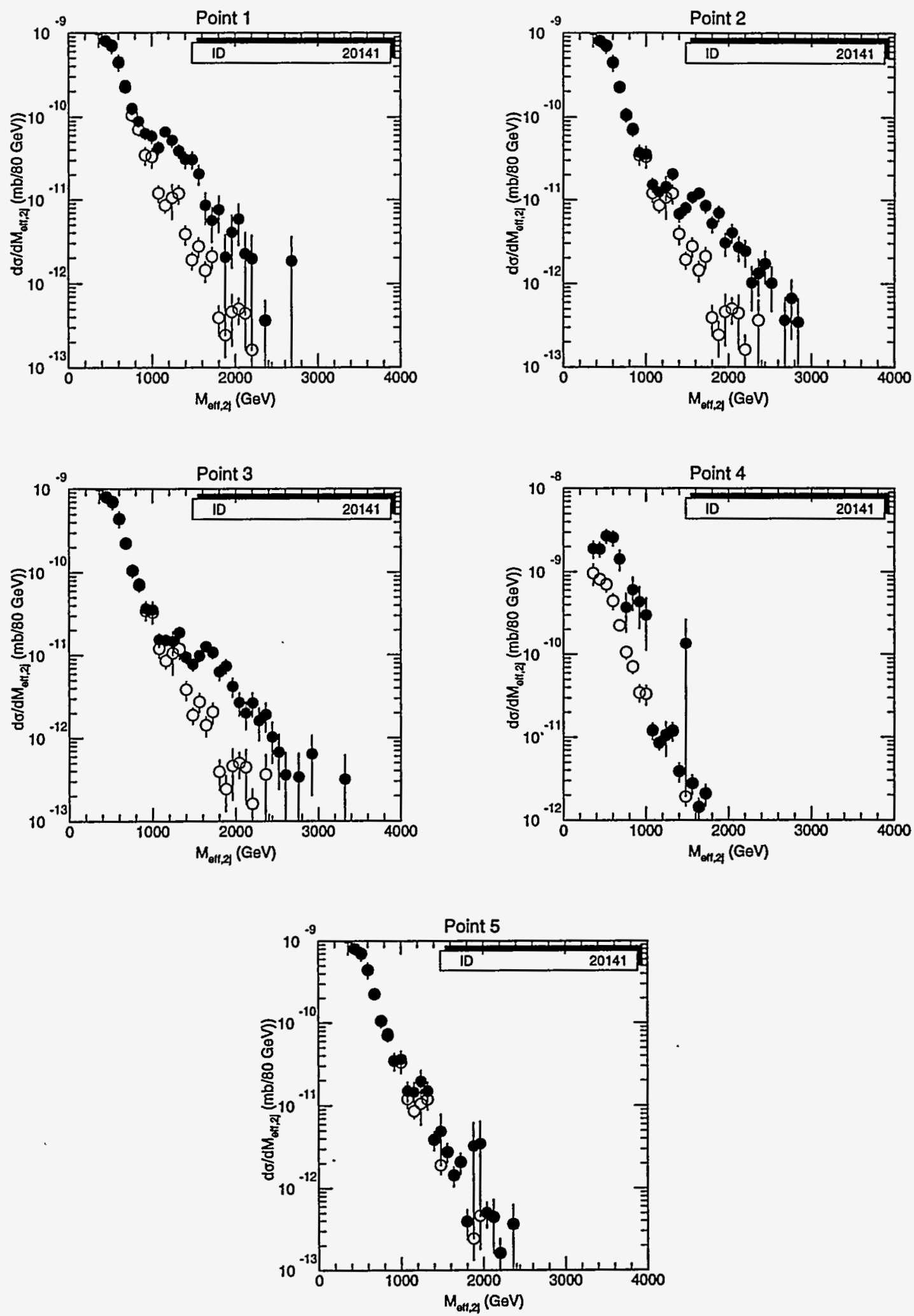

Figure 14: Effective mass $M_{\mathrm{eff}, 2 j}$ distributions. 

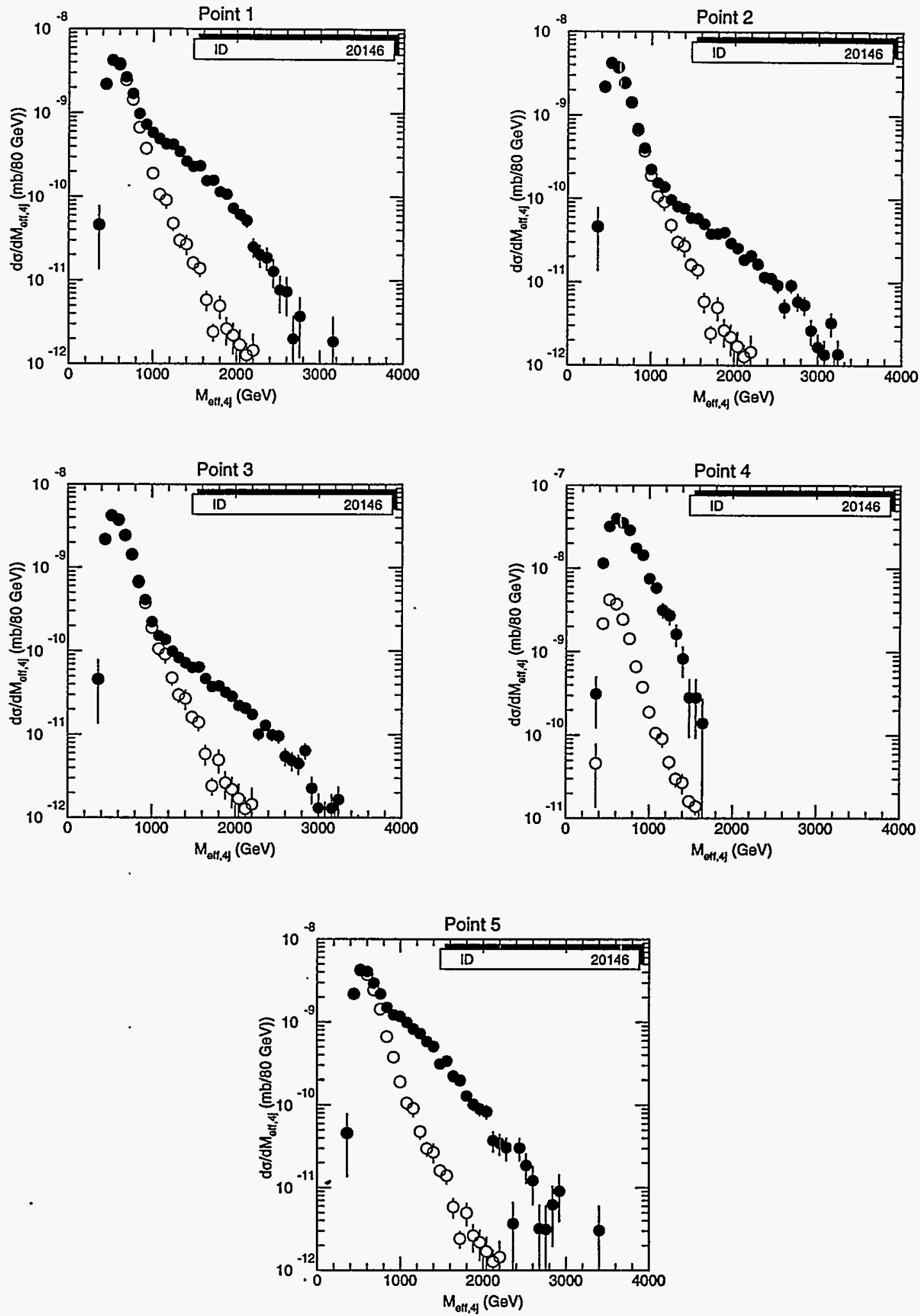

Figure 15: Effective mass $M_{\text {eff }, 4 j}$ distributions. 

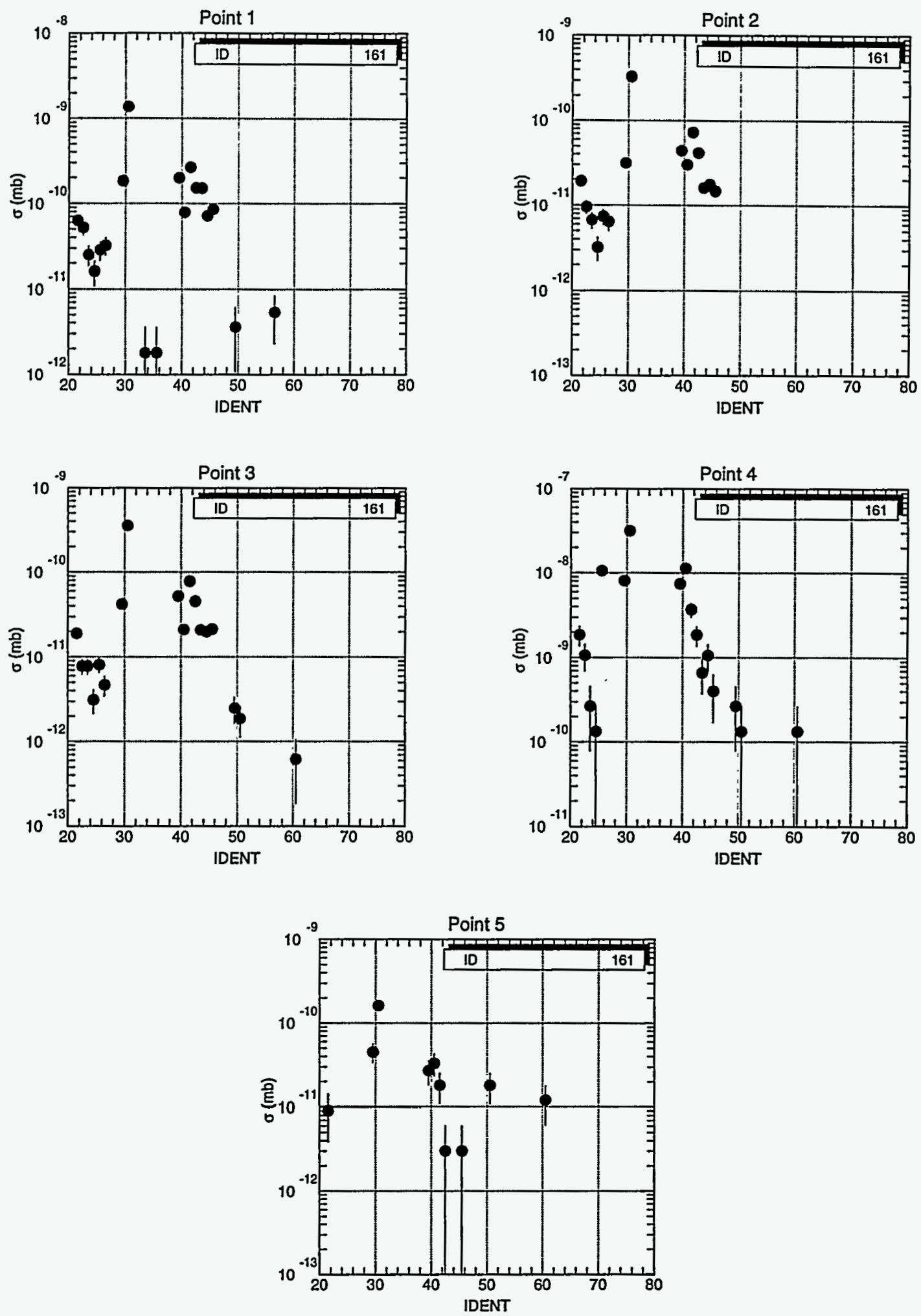

Figure 16: ISAJET types for all SUSY particles in events passing the $M_{\text {eff }, 2 j}$ cuts. See Fig. 1 for notation. 

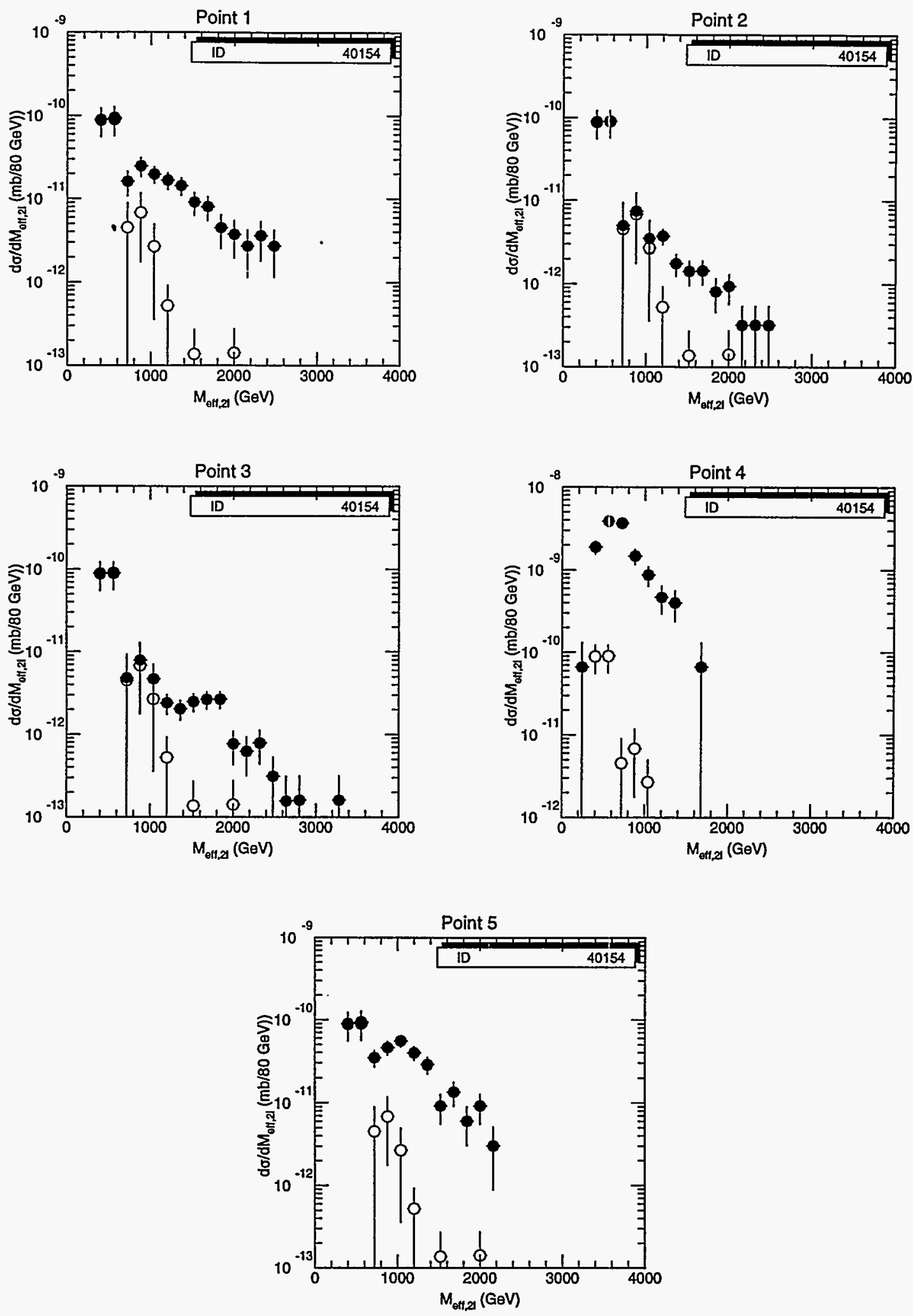

Figure 17: Effective mass $M_{\text {eff, } 2 l}$ distributions for $\ell^{ \pm} \ell^{ \pm}$events. 